Pesq. Vet. Bras. 36(10):1053-1057, outubro 2016 DOI: $10.1590 / \mathrm{S} 0100-736 \mathrm{X} 2016001000020$

\title{
Cortical branches of the middle cerebral artery in silver fox (Vulpes vulpes) $^{1}$
}

\author{
Benedykt Skoczylas², Witold Brudnicki², Krzysztof Kirkiłło-Stacewicz²*, \\ Włodzimierz Nowicki ${ }^{2}$ and Jan Wach ${ }^{2}$
}

\begin{abstract}
Skoczylas B., Brudnicki W., Kirkiłło-Stacewicz K., Nowicki W. \& Wach J. 2016. Cortical branches of the middle cerebral artery in silver fox (Vulpes vulpes). Pesquisa Veterinária Brasileira 36(10):1053-1057. Department of Animal Morphology and Hunting, Faculty of Animal Breeding and Biology, University of Science and Technology, Bernardyńska 6/8, 85-029, Bydgoszcz, Poland. E-mail: krzysztof.stacewicz@o2.pl

The study of the vascularization of the cerebrum in silver fox was performed on 80 cerebral hemispheres. It was found that the middle cerebral artery is the strongest vessel supplying blood to the cerebrum. The artery gets divided into ten permanent branches. Two olfactory arteries supply the region of the cerebrum located on the border between the old and the new cortex. The other eight supply the region of the new cortex. The frontal, parietal and temporal branches descended independently from the main trunk of the middle cerebral artery or formed a common trunk. Common trunks for respective groups of branches have been described as the anterior, superior and posterior middle cerebral artery. The alterior olfactory artery in $5 \%$ of cases and posterior olfactory artery in $2.5 \%$ of cases were independent branches of the middle cerebral artery extending from the rostral cerebral artery.
\end{abstract}

INDEX TERMS: Cerebral artery, silver fox, Vulpes vulpes, brain arteries.

\section{INTRODUCTION}

The first authors investigating the arteries of the brain in animals (Hofmann 1900, Jenke 1919) describe the structure and variability of the brain base arteries. The authors of these works mention only that the middle cerebral artery is one of the cortical branches extending from the arterial circle of the brain. So far, the literature on brain blood flow in silver fox one may find publications on the construction of the brain base arteries (Wiland \& Zawadzińska 1996). The authors of these publications conclude that the middle cerebral artery is one of vessels separating from the arterial circle of the brain. In other predatory species similar study was performed in mink (Wiland 1974), in ferrets (Wiland \& Jablonski 1976) and in raccoon dog (Brudnicki et al. 1994). There are publications in which authors describe the distribution of the middle cerebral artery on specific branches. Cortical branches of the middle cerebral

\footnotetext{
${ }^{1}$ Received on March 29, 2016.

Accepted for publication on July 5, 2016.

${ }^{2}$ Department of Animal Morphology and Hunting, Faculty of Animal Breeding and Biology, University of Science and Technology (UTP), Bernardyńska 6/8, 85-029, Bydgoszcz, Poland. ${ }^{*}$ Corresponding author: krzysztof.stacewicz@o2.pl
}

artery in cat were described by Chadzypanagiotis (1975). Detailed descriptions of the construction and the course of the cortical branches of the middle cerebral artery in some predatory species were presented by Wiland (1991). In recent times, there have been numerous works that discuss the construction of the middle cerebral artery in various mammalian species. This applies to vessels that expand as a single branch e.g. in red squirrel (Aydin 2008), in a the ground squirrel (Aydin et al. 2009), in otter (Skoczylas et al. 2011,2012 ) and multiple arteries in domestic pig (Skoczylas 2000) and hare (Brudnicki et al. 2015). It was found that the cortical branches of the middle cerebral artery in these species come to the same areas of the telencephalon. The differences occur in the pattern of descent and division of respective cortical branches of the middle cerebral artery. The pattern of division of the middle cerebral artery is affected by how the species has been classified and the pattern of groove-coverage of the cortex. In mammals on the surface of the cortex there is a different pattern of sulci, which can affect the structure of the cortical branches of the middle cerebral artery (Brauer \& Schober 1970). In vast literature there seem to be missing a paper on the cortical branches of the middle cerebral artery in silver fox, which is the main blood vessel supplying the cerebrum. Considering 
the discrepancy resulting from respective descriptions and considering new studies, one has decided to investigate the pattern, the division and variation of cortical branches of the middle cerebral artery in silver fox and to compare the results with the data reported by other authors.

\section{MATERIALS AND METHODS}

Ethics statement. The experiment was conducted at Department of Animal Morphology and Hunting, UTP University of Science and Technology, Poland, in compliance with the Ethics Principles in Animal Experimentation, being approved by the Ethics Committee on Animal Experimentation (CEUA/CNPSA) (Protocol \#005/2010).

Study design. The research was performed on 40 brains in silver fox, namely a total of 80 cerebral hemispheres received from a fax farm at Łachowo. The animal heads were cut off at the height of the 3rd to 4th cervical vertebrae. The arteries were filled with latex introduced with medical syringe into the common carotid artery. The heads were fixed in a $5 \%$ formalin solution for 3 months, and then decalcified in hydrochloric acid, the skull cavity was opened and brains were taken out. The cerebral hemispheres were photographed and the following were being described: the anatomy, the division pattern and the course of cortical branches of the middle cerebral artery.

Statistics. Descriptive analysis was performed and the overall result shown in figures.

\section{RESULTS}

In silver fox the blood is supplied to the brain with internal carotid arteries (Fig.1a) and vertebral arteries.

The internal carotid artery, having entered the skull cavity and penetrated the dura mater, bifurcates into the rostral cerebral artery (Fig.1b) and caudal communicating artery (Fig.1c) which, together with their symmetrical vessels form an arterial circle of the brain.

From the initial section of the rostral cerebral artery towards the cortex there separates the middle cerebral artery.

The middle cerebral artery is the strongest vessel supplying blood to the cerebrum. The initial section of the main trunk of the middle cerebral artery goes along the dorsal surface of the optic tract. Then the section gets bended around the piriform lobe and goes through its rostral margin. Further on it runs to the lateral olfactory sulcus and, having passed it, it gets divided. From the initial section of the main trunk of the middle cerebral artery there descend minor central branches supplying blood to olfactory tracts and the piriform lobe. The main trunk of the middle cerebral artery gets divided into a number of cortical branches which run to the specific region of the cerebral hemisphere, supplying blood to specific regions of the brain.

The first permanent branches of the middle cerebral artery which supply both the old and the new cortex are olfactory arteries.

The anterior olfactory artery (Fig.1-1), having separated from the main trunk of the middle cerebral artery, runs to the rostral part of the lateral olfactory sulcus it can ascend into in various places. Its terminal branches can also appear again from under the lateral olfactory sulcus and then ascend under the cortex surface.
The posterior olfactory artery (Fig.1-2) ascends into the caudal part of the lateral olfactory sulcus and its terminal branches supply the area of the cortex found under the sulcus.

The other branches of the middle cerebral artery supply the areas of the cortex over the lateral olfactory sulcus. On the cortex towards the frontal lobe there spread three thick branches. As the first one there separates the orbital branch (Fig.1-3) which is located lowest and it goes towards the region of the Presylvian sulcus where its terminal branches reach the coronary groove.

The inferior frontal branch (Fig.1-4) vascularizes the middle part of the frontal lobe. The vessel goes through the rostral external Sylvian sulcus and the rostral Suprasylvian sulcus towards the coronary groove it passes towards the fornix.

The superior frontal branch (Fig.1-5), having separated from the middle cerebral artery at the height of the rostral external Sylvian sulcus, goes up to the region of the cruciate sulcus. The vessel supplies blood to the upper part of the medial surface of the frontal lobe.

The next vessel which runs towards the parietal lobe bifurcates into two branches.

The anterior parietal branch (Fig.1-6) runs towards the middle external Sylvian sulcus to the marginal sulcus. The terminal twigs of that vessel supply blood to the area of the cortex found under the ansiform sulcus.

The posterior parietal branch (Fig.1-7) also runs to the region of the marginal sulcus and further on it branches out into smaller vessels. Some of them ascend into the medial Suprasylvian sulcus.

The lateral-posterior surface of the cerebral hemisphere is supplied by the branches of the middle cerebral artery which descend from at various heights and they are referred to as temporal branches.

The superior temporal branch (Fig.1-8) is usually the strongest cortical branch of the middle cerebral artery and its further continuation in the area of the cortex. Having left the Sylvian fissure, it runs towards the middle Suprasylvian sulcus and further to the upper margin of the cerebral hemisphere. The branch supplies blood to the upper part of the cortex.

The middle temporal branch (Fig.1-9) descends a small distance away from the previous branch. The branches of that vessel spread towards the external marginal sulcus. Its terminal branches go onto the surface of the occipital lobe.

The inferior temporal branch (Fig.1-10) runs to the end of the caudal external Sylvian sulcus. Having passed the posterior part of the sulcus, its branches spread towards the caudal Suprasylvian sulcus. Its terminal branches take part in the supply of a part of the occipital lobe.

Considering the general pattern of the spread the cortical branches of the middle cerebral artery in silver fox, one shall note that respective sections of those branches can run inside respective sulci, sometimes undergoing further divisions, always running towards the cortex areas described. Analysing the pattern of descent of the cortical branches of the middle cerebral artery in the silver fox 
individuals investigated, it was found that from the rostral cerebral artery on 74 (92.5\%) cerebral hemispheres there descended a single independent vessel; the middle cerebral artery. Among them on 16 (20\%) hemispheres from the main trunk of the middle cerebral artery there descended rostrally a common trunk for the anterior olfactory artery and for the orbital branch, then a common descend from the inferior and superior frontal branches. The main trunk caudally gave rise to the posterior olfactory artery with a common descent with the anterior temporal branch. Having ascended into the Sylvian fissure, on the surface of the cortex it showed a common trunk for anterior and posterior parietal branches as well as for the middle and superior temporal branches.

In another 8 (10\%) cases there descended rostrally an independent anterior olfactory artery and a common trunk for the orbital, anterior and superior frontal branches. The main trunk got onto the surface of the cerebral cortex from the Sylvian fissure and formed a common descent for anterior and posterior parietal branches. Caudally from the main trunk of the middle cerebral artery, with a common trunk there separated the superior, middle and inferior temporal branches, whereas the posterior olfactory artery got separated independently from the main trunk of the middle cerebral artery.

On another 14 (17.5\%) hemispheres from the main trunk the following separated rostrally with a common trunk: the orbital branch, the inferior frontal branch and the anterior olfactory artery. The caudal branch was the common trunk for inferior temporal branch and posterior olfactory artery. The main trunk, having ascended into the Sylvian fissure, on the surface of the cortex it showed a common trunk for superior frontal branch, anterior and posterior parietal branches as well as for the middle and superior temporal branches.

On another 12 (15\%) hemispheres from the main trunk the following separated rostrally with a common trunk: the anterior olfactory artery, common trunk for the orbital branch, the inferior and superior frontal branch. The main trunk of the middle cerebral artery, having got into the surface of the cortex, separated a common descent for anterior and posterior parietal branches. Caudally from the main trunk the following separated with a common descent: the inferior, middle and superior temporal branches as well as an independent posterior olfactory artery.

On yet another 10 (12.5\%) cerebral hemispheres from the main trunk rostrally there separated, with a common descent, the orbital branch, the inferior frontal branch and the anterior olfactory artery. The main trunk of the middle cerebral artery, having got into the surface of the cortex, gave a common descent for the superior frontal branch, anterior and posterior parietal branches. Caudally from the main trunk the following separated with a common descent: the inferior, middle and superior temporal branches as well as an independent posterior olfactory artery.

On another 14 (17.5\%) cerebral hemispheres from the main trunk of the middle cerebral artery departed rostrally the common trunk for the anterior olfactory artery and for the orbital branch, then a common departure for the inferior and superior frontal branches. Caudally from the main trunk there descended the inferior olfactory artery through the common trunk with the inferior temporal branch. The main trunk, having descended into the Sylvian fissure, got onto the surface of the cortex with a common descent for anterior and posterior parietal branches as well as the middle and superior temporal branch.

On the other $6(7,5 \%)$ hemispheres it was found that

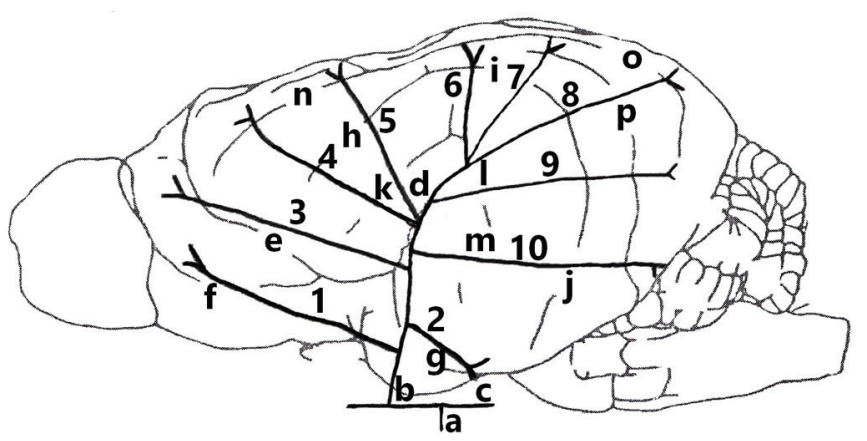

Fig.1. Diagram of the division of the middle cerebral artery on the surface of the cortex in silver fox. Anterior olfactory artery (1), posterior olfactory artery (2), orbital branch (3), inferior frontal branch (4), superior frontal branch (5), anterior parietal branch (6), posterior parietal branch (7), superior temporal branch (8), middle temporal branch (9), inferior temporal branch (10). Internal carotid artery (a), rostral cerebral artery (b), caudal communicating artery (c), Sylvian fissure (d), Presylvian sulcus (e), rostral lateral olfactory sulcus (f), caudal lateral olfactory sulcus (g), rostral Suprasylvian sulcus (h), middle Suprasylvian sulcus (i), caudal Suprasylvian sulcus (j), rostral external Sylvian sulcus (k), middle external Sylvian sulcus (l), caudal external Sylvian sulcus (m), coronary sulcus (n), marginal sulcus (o), external marginal sulcus (p).

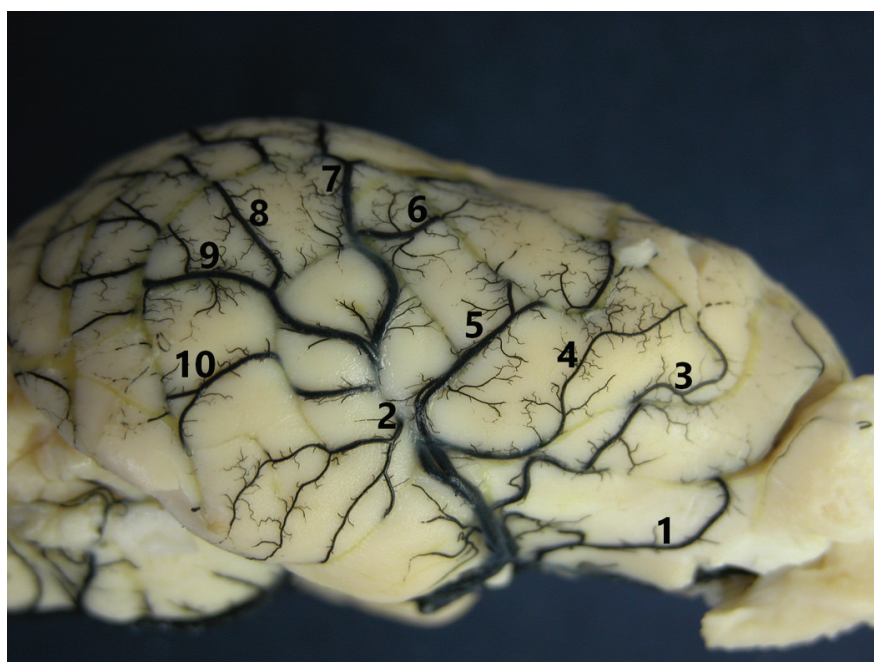

Fig.2. Independent departure of the alterior olfactory artery and the main trunk of the middle cerebral artery from the rostral cerebral artery - right side. Anterior olfactory artery (1), posterior olfactory artery (2), orbital branch (3), inferior frontal branch (4), superior frontal branch (5), anterior parietal branch (6), posterior parietal branch (7), superior temporal branch (8), middle temporal branch (9), inferior temporal branch (1). 


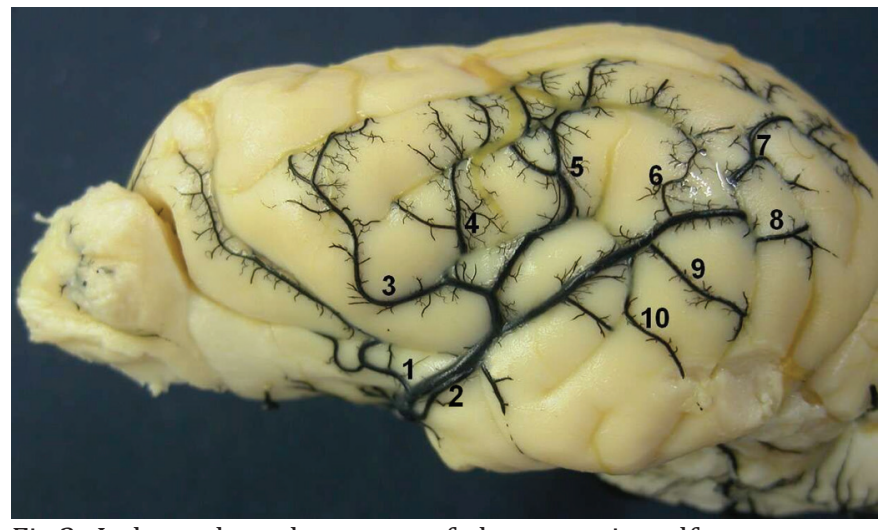

Fig.3. Independent departure of the posterior olfactory artery from the rostral cerebral artery (left side). Anterior olfactory artery (1), posterior olfactory artery (2), orbital branch (3), inferior frontal branch (4), posterior frontal branch (5), parietal branches $(6,7)$, temporal branches $(8,9,10)$.

from the rostral cerebral artery there bifurcated two independent branches of the middle cerebral artery. Among them in 4 (5\%) cases the first independent branch from the rostral cerebral artery was the anterior olfactory artery, while the second branch from the rostral cerebral artery - the main trunk of the middle cerebral artery from which there descended rostrally independently: the orbital branch, the inferior and superior frontal branch. Caudally from the main trunk there separated an independent posterior olfactory artery and the inferior temporal branch. The main trunk, having descended into the Sylvian fissure, got onto the surface of the cortex with a common descent for anterior and posterior parietal branches as well as the middle and superior temporal branch (Fig.2).

On yet another $2(2.5 \%)$ brains from the rostral cerebral artery there descended independently the posterior olfactory artery. The other independent descent from the rostral cerebral artery was the main trunk of the middle cerebral artery from which, rostrally and independently there separated the alterior olfactory artery and the common trunk for the inferior and superior frontal branch and the orbital branch. Caudally from the main trunk there separated the common trunk for the inferior and superior temporal branch. The main trunk, having ascended into the Sylvian fissure, got onto the cortex surface with the anterior and posterior parietal branches as well as the superior temporal branch (Fig.3).

\section{DISCUSSION}

The middle cerebral artery supplies blood to the greatest region of the cerebrum. In the silver fox the middle cerebral artery supplies the same areas of the brain as in the mammalian species studied so far. The discrepancies concern mostly its division into respective branches. Chadzypanagiotis (1975), describing the cortical branches in cat, differentiated between the branches supplying the old cortex, the branches on the border of the old and the new cortex as well as the branches for the new cortex. In the silver fox the arteries supplying the old cortex are minor branches onto the piriform lobe and olfactory tracts.
On the border of the old and the new cortex there are found the anterior and posterior olfactory arteries. In the silver fox the anterior olfactory artery in 5\% of the cases was a vessel which descended independently from the rostral cerebral artery. On the other cerebral hemispheres it was a vessel which got separated independently from the main trunk of the middle cerebral artery in $37.5 \%$ of the cases. On the $30 \%$ of the cerebral hemispheres it was one of the branches descending from the common trunk of the middle cerebral artery which gave rise to the orbital branch and the inferior frontal branch. In the other $10 \%$ cases the anterior olfactory artery demonstrated a common descent with the orbital, inferior and superior frontal branches.

The posterior olfactory artery, on the other hand, in $2.5 \%$ of the cases was a vessel which descended independently from the rostral cerebral artery. On $42.5 \%$ hemispheres it was the vessel descending independently from the main trunk of the middle cerebral artery. In $27.5 \%$ of the cases the posterior olfactory artery separated with a common descent with the inferior temporal branch. In the other 35\% hemispheres the posterior olfactory artery was one of the branches of a common trunk for inferior temporal branch. In another $20 \%$ of the cases it was one of the branches of the common trunk for the inferior and middle temporal branches.

The other cortical branches of the middle cerebral artery can be divided into a group of frontal, parietal and temporal branches. In the silver fox, similarly as in other Carnivora species there occur eight main vessels which supply blood to the area of the new cortex of the cerebrum.

Besides, respective cortical branches can descend from the main trunk of the middle cerebral artery with a common descent. Such cases of descent were reported by Wiland (1991), Skoczylas et al. (2012) as the anterior, superior and posterior middle cerebral artery. In silver fox the anterior middle cerebral artery has been presented as a common trunk for frontal branches and it occurred in 35\% of the cases investigated, the superior middle cerebral artery was described as a common trunk for parietal branches, which was observed in $15 \%$ of the cases. The posterior middle cerebral artery as a common trunk for temporal branches was found in $27,5 \%$ of the cases.

In silver fox the superior middle cerebral artery occurred as the lowest percentage of the cases, however, here the anterior middle cerebral artery dominated. Making a comparison of the present results with those reported by Skoczylas et al. (2012), one can state that also in otter the superior middle cerebral artery was reported as the lowest percentage of the cases. In silver fox, similarly as in the other animal species studied, the parietal branches have developed poorest. On the surface of the cerebrum the best developed are the frontal branches of the middle cerebral artery.

From the description of the structure of the middle cerebral artery in the publications by Aydin (2008), Skoczylas et al. (2012), Ozudogru et al. (2012) in the red squirrel, fallow deer, common fox and cattle (Skoczylas et al. 2016) one can see that it is usually a single vessel descending from the rostral cerebral artery. The vessel, 
having passed the lateral olfactory sulcus, gets divided along its course into respective cortical branches. In the material investigated such a pattern of division of the middle cerebral artery was found in $92.5 \%$ of the cases. In silver fox there were identified the cases of descent from the rostral cerebral artery of two independent arterial trunks in 7,5\% of the cases. The second independent branch from the rostral cerebral artery was the anterior olfactory artery in $5 \%$ of the cases, the posterior olfactory artery $-2.5 \%$ of the cases and a common trunk of the anterior olfactory artery with the orbital branch in $2.5 \%$ of the cases. In other mammalian species the presence of two independent descents of the branches of the middle cerebral artery was found in raccoon dog (Brudnicki et al. 1994) in 18,6\% of the cases, Skoczylas et al. 2015 - in 5\% of cases and in wild rabbit (Brudnicki et al. 2012) in 36.5\% of the cases.

The present research show that observed in silver fox the division of the middle cerebral artery into the same branches or their groups, like in the other mammalian species investigated so far is a result of genetic limitations. According to Wiland (1980), in the blood supply of the brain in individuals of particular species, the resource of genetic information accumulated during the phylogenetic development is essential.

\section{CONCLUSIONS}

The division of the middle cerebral artery into the same branches or their groups is a result of genetic limitations.

The alterior olfactory artery in 5\% of cases and posterior olfactory artery in $2.5 \%$ of cases were independent branches of the middle cerebral artery extending from the rostral cerebral artery.

In silver fox the superior middle cerebral artery occurred as the lowest percentage of the cases, however, here the anterior middle cerebral artery dominated.

The middle cerebral artery is the strongest vessel supplying blood to the cerebrum and gets divided into ten permanent branches.

Two olfactory arteries supply the region of the cerebrum located on the border between the old and the new cortex.

The other eight supply the region of the new cortex.

Conflict of interest statement.- The authors have no competing interests.

\section{REFERENCES}

Aydin A. 2008. The morphology of circulus arteriosus cerebri in the red squirrel (Sciurus vulgaris). Veterinarni Medicina 53(5):272-276.
Aydin A., Ozkan Z.E., Yilmaz S. \& Ilgun R. 2009. The morphology of the circulus arteriosus cerebri in the ground squirrel (Spermophilus citellus). Veterinarni Medicina 54(11):537-542.

Brauer K. \& Schaber W. 1970. Katalog der sangetiergehirne, VEB Gustaw Fisher Verlag, Jena.

Brudnicki W., Wiland C. \& Jabłoński R. 1994. Basilar arteries of the brain in raccoon dog (Nyctereutes procyonoides Gray). Archiv Vet. Pol. 34(1/2):141-147.

Brudnicki W., Nowicki W., Skoczylas B., Brudnicki A., Kirkiłło-Stacewicz K. \& Wach J. 2012. Arteries of the brain in wild European rabbit Oryctolagus cuniculus (Linnaeus, 1758). Folia Biologica 60(3/4):189-194.

Brudnicki W., Kirkiłło-Stacewicz K., Skoczylas B., Nowicki W., Jabłoński R., Brudnicki A. \& Wach J. 2015. The arteries of the brain in hare (Lepus europaeus Pallas, 1778). The Record 298:1774-1779.

Chadzypanagiotis D. 1975. Arteries on the surface of the cerebral hemisphere in the cat. Folia Morphologica, Warsaw, 32:385-399.

Hofmann M. 1900. Zur vergleichenden Anatomie der Gehirn und Rückenmarksarterien der Vertebraten. Z. f. Morphol. u. Anthropol. 2:247-320.

Jenke T.W. 1919. Die Gehirnarterien des Pferdes, Hundes, Rindes und Schweines verglichen mit denen des Menschen. Diss. Dresden.

Ozudogru Z., Can M. \& Balkaya H. 2012. Macro-Anatomical Investigation of the Cerebral Arterial Circle (Circle of Willis) in Red Fox (Vulpes vulpes Leunnoleus, 1758). J. Anim. Vet. Advances 11(16):2861-2864.

Skoczylas B. 2000. Cortical branches of middle cerebral artery in domestic pig (Sus scrofa f. domestica). EJPA Univ. Vet. Med. 3, Issue: 1-6.

Skoczylas B., Brudnicki W., Nowicki W., Jabłoński R., Kirkiłło-Stacewicz K. \& Wach J. 2011. Cortical branches of the middle cerebral artery in fallow deer (Dama dama). EJPAU, Seria Vet. Med. 14(4):9.

Skoczylas B., Brudnicki W., Nowicki W., Kirkiłło-Stacewicz K., Jabloński R. \& Wach J. 2012. The cortical branches of the middle cerebral artery in the otter (Lutra lutra). Veterinarni Medicina 57(6):282-286.

Skoczylas B., Brudnicki W., Kirkiłło-Stacewicz K., Nowicki W. \& Wach J. 2015. Telencephalon vascularity in raccoon dog (Nyctereutes Proteyonoides grey (1834). EJPAU 18(1), \#4.

Skoczylas B., Brudnicki W., Kirkiłło-Stacewicz K., Nowicki W. \& Wach J. 2016. Arterial supply of the cerebral cortex in cattle (Bos primigenius $\mathrm{f}$. domesticus). Slovenian Vet. Res. 53(1):13-18.

Wiland C. 1974. Variability of the brain base arteries and aortic arch in American mink. BTN - The Papers of the Commission for Natural Sciences B 20:79-108.

Wiland C. 1980. Variability of the brain base arteries in the populations of species of the family Canidae and Mustelidae. BTN, The Papers of the Department of Natural Sciences B 29:73-92.

Wiland C. 1991. Comparative studies of cortical branches of the middle cerebral artery in some predatory species (Carniviora). Scientific Journals ATR Bydgoszcz 44:1-52.

Wiland C. \& Jabłoński R. 1976. Variation of the basal arteries of the brain in polecat (Mustela putorius putorius L.). Anatomischer Anzeiger 140:498-506.

Wiland C. \& Zawadzińska B. 1996. The case of vascular variety in the arterial circle of the brain in silver fox. Scientific Journals ATR Bydgoszcz Zoot. 38:35-40. 